\title{
Fluid-structure interaction of two bodies in an inviscid fluid
}

\author{
A. A. Tchieu, ${ }^{1, a)}$ D. Crowdy, ${ }^{2}$ and A. Leonard ${ }^{1}$ \\ ${ }^{1}$ Graduate Aerospace Laboratories, California Institute of Technology, \\ 1200 E. California Boulevard, Pasadena, California 91125, USA \\ ${ }^{2}$ Department of Mathematics, Imperial College London, \\ 180 Queen's Gate, London SW7 2AZ, United Kingdom
}

(Received 4 March 2010; accepted 11 August 2010; published online 1 October 2010)

\begin{abstract}
The interaction of two arbitrary bodies immersed in a two-dimensional inviscid fluid is investigated. Given the linear and angular velocities of the bodies, the solution of the potential flow problem with zero circulation around both bodies is reduced to the determination of a suitable Laurent series in a conformally mapped domain that satisfies the boundary conditions. The potential flow solution is then used to determine the force and moment acting on each body by using generalized Blasius formulas. The current formulation is applied to two examples. First, the case of two rigid circular cylinders interacting in an unbounded domain is investigated. The forces on two cylinders with prescribed motion (forced-forced) is determined and compared to previous results for validation purposes. We then study the response of a single "free" cylinder due to the prescribed motion of the other cylinder (forced-free). This forced-free situation is used to justify the hydrodynamic benefits of drafting in aquatic locomotion. In the case of two neutrally buoyant circular cylinders, the aft cylinder is capable of attaining a substantial propulsive force that is the same order of magnitude of its inertial forces. Additionally, the coupled interaction of two cylinders given an arbitrary initial condition (free-free) is studied to show the differences of perfect collisions with and without the presence of an inviscid fluid. For a certain range of collision parameters, the fluid acts to deflect the cylinder paths just enough before the collision to drastically affect the long time trajectories of the bodies. In the second example, the flapping of two plates is explored. It is seen that the interactions between each plate can cause a net force and torque at certain instants in time, but for idealized sinusoidal motions in irrotational potential flow, there is no net force and torque acting at the system center. (C) 2010 American Institute of Physics. [doi:10.1063/1.3485063]
\end{abstract}

\section{INTRODUCTION}

This paper addresses the problem of solving for the hydrodynamic interactions between two arbitrarily moving rigid bodies in an inviscid fluid. Not only is it of theoretical interest, but this work also is important for the application to a myriad of doubly connected problems. As an example of such a doubly connected domain problem, the Weis-Fogh mechanism has been previously studied by using complex potential theory for the spreading phase until the two wings separate into an inherently two-body problem. ${ }^{1}$ The analysis within this study does not carry on into the phase where the wings are separated due to a lack of a generalized potential theory for doubly connected domains. Similarly, if we examine the cross-section of a dragonfly wing, we see two wings that constitute another naturally occurring two-body problem where the interactions between each wing cannot be simply neglected. ${ }^{2}$ To the authors' knowledge, the hydrodynamics of such phenomena have not been previously investigated and the framework provided in this paper will allow its subsequent analysis. Moreover, another relevant example in aerodynamics concerns the flow around two airfoils, or the socalled "biplane problem," where it has been seldom studied from an analytic standpoint. In particular, Sedov's ${ }^{3}$ work on this subject is a rare resource and he devotes a short chapter

${ }^{a)}$ Electronic mail: atchieu@ gmail.com. to determining the steady flow around several biplane sections in his book. Many other examples of situations with fundamentally doubly connected domains exist and the reader is urged to examine Refs. 4 and 5 for applications to turbomachinery and coordinated motion applied to fish schooling, respectively.

Until recently, the multiply connected domain problem in potential theory has been avoided except for a number of simplified cases. Perhaps as the simplest case, Lagally ${ }^{6}$ was the first to consider the analytical solution of the potential flow problem for two stationary cylinders in a uniform stream. He obtained a rather complicated expression for the velocity potential in terms of Weierstrass functions. This work was later extended to the case of two hydrodynamically coupled cylinders by Landweber and Shahshalan ${ }^{7}$ to study the hydrodynamic forces on two bodies nearing inline impact. Furthermore, Wang ${ }^{8}$ contributed to this specific problem by determining the forces on the cylinders with the extension of allowing the cylinders to contract and expand, as well as determining the large distance influence of the cylinders on one another. More recently, Borisov ${ }^{9}$ provided the first dynamically coupled interaction between two circular cylinders in a perfect fluid and restricted his examples to the motion of his cylinders to be along the line connecting their centers. We also take notice in the long-lasting interest in the two cylinder problem, as Alassar ${ }^{10}$ has recently revisited the 
fundamental question proposed by Lagally and determined an alternative solution to the potential flow problem using bipolar coordinates and an infinite series expansion. The methods referenced above are very restrictive in the sense that they are only applicable to the case where the bodies are two circular cylinders.

For more complicated shapes, the use of boundary integral formulations, such as panel methods (for example, see Ref. 11), have supplanted closed-form solutions for the potential due to the lack of a theory. However, Crowdy ${ }^{12}$ has developed a new calculus to address issues with providing solutions to flows in multiply connected domains and has applied it to the Weis-Fogh mechanism discussed above. ${ }^{13}$ Although the doubly connected problem can be solved in Crowdy's framework, here we provide an efficient and specific solution of the flow generated by the motion of two arbitrarily shaped bodies without the need of special functions. This stems from the fact that the doubly connected case has special properties not available in the general multiply connected case, which are exploited in order to avoid using the integral representation by Crowdy. ${ }^{12}$ In addition, formulas for the force and moment are provided and a method to study the fluid-structure interaction of the system is proposed.

As a specific example, the interaction of two circular cylinders is more closely investigated using the proposed method. First, the method is validated by determining the hydrodynamic forces for the prescribed motions of the two cylinders and comparing the results to previously derived formulations in Wang, ${ }^{8}$ Bampalas and Graham, ${ }^{14}$ and Landweber and Shashalan. ${ }^{7}$ Next, numerical analyses are performed to address the benefits of hydrodynamic drafting in a "forced-free" interaction by prescribing the motion of one cylinder and allowing the second cylinder to follow freely. Lastly, analysis of "free-free" interaction of two cylinders is carried out. Here, the idea of free-free interactions refers to objects that are given initial conditions and allowed to interact with the flow field freely, which in part has been studied by Borisov ${ }^{9}$ for specific inline motions of the cylinders. Here, we choose to concentrate on studying the oblique collision and near-collision events of two hydrodynamically coupled cylinders and demonstrate that the system conserves total energy and momentum.

An additional example on two flapping plates similar to the configuration discussed in $\mathrm{Wang}^{2}$ is provided to highlight the use of the method in a more complicated doubly connected domain. An idealized flapping motion is examined and resulting forces and torque on both individual plates are determined for the specified motion.

\section{POTENTIAL FLOW FORMULATION}

\section{A. Formulation of the boundary value problem}

We seek an incompressible, inviscid, and irrotational flow in an unbounded two-dimensional fluid domain $D$ that is exterior to two solid objects, $D_{1}$ and $D_{2}$. Let us denote each body by a subscript $k=1,2$ so that each body moves with velocity $\mathbf{U}_{k}=\left(u_{k}, v_{k}\right)$ and rotates with the angular rate $\Omega_{k}$. Kelvin's theorem guarantees that a flow that originates in an irrotational flow must remain irrotational everywhere; thus, for all times there exists a velocity potential $\Phi$ such that $\mathbf{u}=\nabla \Phi$. Incompressibility requires that the velocity potential must satisfy Laplace's equation $\nabla^{2} \Phi=0$ in the domain $D$. Since $\Phi$ is harmonic, the solution can additionally be recast as finding its harmonic conjugate, the stream function $\Psi$. Additionally, it is assumed that the flow decays at infinity and that the circulation around each object is zero. The solution must satisfy the boundary conditions

$$
\mathbf{u} \cdot \hat{\mathbf{n}}=\left[\mathbf{U}_{k}+\Omega_{k} \hat{\mathbf{e}}_{z} \times\left(\mathbf{x}-\mathbf{x}_{k}\right)\right] \cdot \hat{\mathbf{n}}, \quad \text { on } \partial D_{k}
$$

for $k=1,2$, where $\hat{\mathbf{n}}$ denotes the unit outward facing normal. In what follows, it is understood that the boundary conditions are, for each body, $k=1,2$. We introduce $s$ to denote the length along a specific contour $\partial D_{k}$, such that the tangent vector and outward normal can be written as

$$
\hat{\mathbf{s}}=\left(\frac{\mathrm{d} x}{\mathrm{~d} s}, \frac{\mathrm{d} y}{\mathrm{~d} s}\right)
$$

and

$$
\hat{\mathbf{n}}=\left(\frac{\mathrm{d} y}{\mathrm{~d} s},-\frac{\mathrm{d} x}{\mathrm{~d} s}\right) .
$$

By the Cauchy-Riemann equations, it is recognized that the normal velocity on the surface $\mathbf{u} \cdot \hat{\mathbf{n}}=\nabla \Phi \cdot \hat{\mathbf{n}}=\nabla \Psi \cdot \hat{\mathbf{s}}$. Therefore Eq. (1), by using definitions (2) and (3), can be rewritten in terms of the stream function as

$$
\begin{aligned}
\frac{\partial \Psi}{\partial x} \frac{\mathrm{d} x}{\mathrm{~d} s}+\frac{\partial \Psi}{\partial y} \frac{\mathrm{d} y}{\mathrm{~d} s}= & -\left[v_{k}+\Omega_{k}\left(x-x_{k}\right)\right] \frac{\mathrm{d} x}{\mathrm{~d} s} \\
& +\left[u_{k}-\Omega_{k}\left(y-y_{k}\right)\right] \frac{\mathrm{d} y}{\mathrm{~d} s}
\end{aligned}
$$

on $\partial D_{k}$. For an arbitrary surface, Eq. (4) is satisfied only if

$$
\begin{aligned}
& \frac{\partial \Psi}{\partial x}=-v_{k}-\Omega_{k}\left(x-x_{k}\right), \quad \text { on } \partial D_{k}, \\
& \frac{\partial \Psi}{\partial y}=u_{k}-\Omega_{k}\left(y-y_{k}\right), \quad \text { on } \partial D_{k} .
\end{aligned}
$$

Upon integration of Eq. (5), $\Psi$ on the boundary is

$\Psi=u_{k} y-v_{k} x-\Omega_{k}\left(\frac{x^{2}+y^{2}}{2}-x_{k} x-y_{k} y\right)+d_{k}, \quad$ on $\partial D_{k}$,

where $d_{k}$ are constants of integration.

Now consider the complex variable $z=x+i y$, such that there exists a complex potential function $W(z)=\Phi+i \Psi$ that is analytic in the domain $D$. In addition, we denote the object velocity in complex form as $U_{k}=u_{k}+i v_{k}$. The problem is now presented in the complex $z$-plane in Fig. 1. Equation (6) can now be rewritten in complex form to read

$$
\operatorname{Im}[W(z)]=f(z)+d_{k}, \quad \text { on } \partial D_{k},
$$

where 

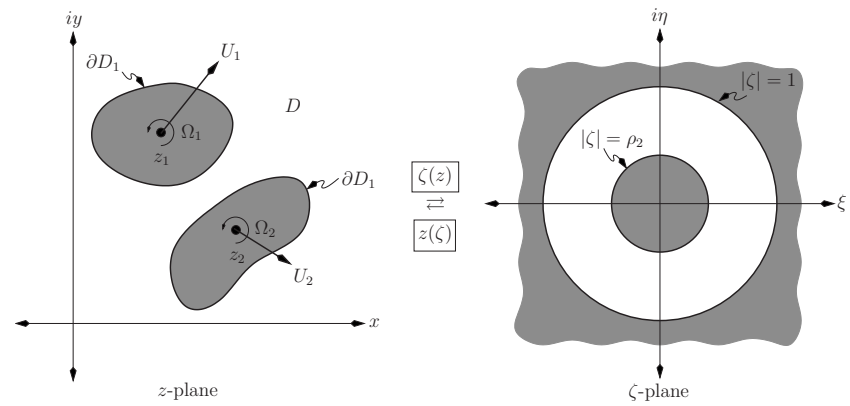

FIG. 1. Two arbitrarily shaped objects in the z-plane mapped to the annulus in the $\zeta$-plane. Object $k$ translates with complex velocity $U_{k}$ and rotates with angular velocity $\Omega_{k}$ around its center of inertia $z_{k}$. The annulus is centered at $\zeta=0$ with a conformal modulus of $\rho_{2}$.

$$
f(z)=\operatorname{Im}\left[\bar{U}_{k} z-i \Omega_{k}\left(\frac{1}{2} z \bar{z}-z \bar{z}_{k}\right)\right] .
$$

Here, "Im" denotes the imaginary part ("Re" the real part) and the overbar denotes explicit complex conjugation. The set of equations for each $k$ in Eq. (7) is equivalent to the standard mathematical problem in potential theory known as the modified Schwarz problem. ${ }^{15}$ Simply stated, if one knows either the real or imaginary value of the complex function on the boundaries, then an analytic solution for $W(z)$ can be found in the domain $D$. Without loss of generality, the constant $d_{1}$ can be set to zero since it can be arbitrarily specified due to the nature of the modified Schwarz problem. The constant $d_{2}$ on the other hand must be determined by a compatibility condition that forces $W(z)$ to be single valued, as discussed later in Sec. II B.

To make further progress, an extension of the Riemann mapping theorem is employed to doubly connected domains. The theorem states that any doubly connected domain can be mapped, by some conformal map $z(\zeta)$, from the annulus given by $\rho_{2}<|\zeta|<1$ in the $\zeta$-plane, where $\rho_{2}$ is defined by the choice of domain $D$ and its appropriate mapping (see Fig. 1). ${ }^{16}$ Defining

$$
w(\zeta)=W[z(\zeta)],
$$

Eq. (7) can be recast as a boundary value problem for $w(\zeta)$ on the annulus in the $\zeta$-plane, i.e.,

$$
\operatorname{Im}[w(\zeta)]=f[z(\zeta)]+d_{k}, \quad \text { on }|\zeta|=\rho_{k},
$$

where $\rho_{1}=1$. It is important to observe that due to the geometric relationship $\bar{\zeta}=\rho_{k}^{2} / \zeta$ on the boundary, the function $f[z(\zeta)]$ in Eq. (9) can be written as an analytic function of $\zeta$ on the boundaries. Additionally, as $z \rightarrow \infty$ the potential must decay to a constant, at most. Therefore, this sets a requirement on the mapped potential such that $w\left(\zeta_{\infty}\right)$ must be bounded, where the preimage of infinity is defined as the $\lim _{\zeta \rightarrow \zeta_{\infty}} z(\zeta)=\infty$.

We note in passing that Eq. (9) is essentially equivalent to the formulation given in Crowdy et al. [see Eq. (3) in Ref. 17] since $\operatorname{Im}[W(z)]=\operatorname{Re}[-i W(z)]$. In contrast to Crowdy et al., ${ }^{17}$ the rotations $\Omega_{k}$ are introduced as additional degrees of freedom in the current formulation.

\section{B. Analytic solution to the boundary value problem}

Although the solution for $w(\zeta)$ can be obtained by using the Villat formula (see Refs. 17-19), we prefer here to use a representation of the complex potential in terms of a Laurent series because it is a natural representation of the solution in the annulus domain. By using the Laurent series expansion, the determination of the Laurent coefficients will stem directly from the application of the boundary conditions. One reason for using this method is that, as shown below, the values of the coefficients can be efficiently calculated by using fast Fourier transforms. Thus, consider the Laurent series expansion of the potential

$$
w(\zeta)=\sum_{j=-\infty}^{\infty} a_{j} \zeta^{j}
$$

The representation given in Eq. (10) satisfies the condition at infinity as long as the series is convergent. To determine the coefficients $a_{j}$, the required boundary data in Eq. (9) are expanded and rewritten as an analytic function, i.e.,

$$
\begin{aligned}
\operatorname{Im}[w(\zeta)] & =\frac{1}{2 i}\left[w(\zeta)-\bar{w}\left(\rho_{k}^{2} \zeta^{-1}\right)\right], \quad \text { on }|\zeta|=\rho_{k}, \\
& =\frac{1}{2 i} \sum_{j=-\infty}^{\infty}\left(a_{j}-\bar{a}_{-j} \rho_{k}^{-2 j}\right) \zeta^{j}, \quad \text { on }|\zeta|=\rho_{k},
\end{aligned}
$$

where the overbar on $w$ denotes the Schwarz conjugate of the function

$$
\bar{w}(\zeta)=\overline{w(\bar{\zeta})} .
$$

Subsequently, the known boundary data are also expanded as

$$
f[z(\zeta)]+d_{k}=\sum_{j=-\infty}^{\infty} b_{k, j} \zeta^{j}+d_{k}, \quad \text { on }|\zeta|=\rho_{k},
$$

where (see Ref. 20, p. 269)

$$
b_{k, j}=\frac{1}{2 \pi i} \oint_{|\zeta|=\rho_{k}} \frac{f[z(\zeta)]}{\zeta^{j+1}} \mathrm{~d} \zeta .
$$

The coefficients (14) can be solved explicitly because the integrand can be written as an analytic function on the boundary. To do so, one can use residue theory for every $j$ th term. Practically, due to the duality of the Laurent series and the Fourier transform, the coefficients (14) can be calculated numerically in an efficient manner by using the fast Fourier transform. The Laurent coefficients can be found from the Fourier series representation of the boundary data by relating $b_{k, j}^{\prime}=b_{k, j} \rho_{k}^{j}$, where $b_{k, j}^{\prime}$ are the Fourier coefficients. It is also useful to recognize that due to the fact that real data are provided on the boundary for the determination of these coefficients, the negative coefficients are given by the alternative relationship $b_{k,-j}=\rho_{k}^{2 j} \bar{b}_{k, j}$ for $j \geq 0$.

Equating coefficients in Eqs. (11) and (13) leads to

$$
a_{j}=\frac{2 i}{1-\rho_{2}^{2 j}} \begin{cases}b_{1, j}-b_{2, j} \rho_{2}^{2 j}, & j>0, \\ b_{2, j}-b_{1, j} \rho_{2}^{2 j}, & j<0 .\end{cases}
$$

The zero term must be explicitly treated as a compatibility constraint. Equating the constant terms uniquely defines 
$d_{2}=b_{1,0}-b_{2,0}$ in addition to requiring $\operatorname{Im}\left[a_{0}\right]=b_{1,0}$. It is observed that the solution for $w(\zeta)$ can only be solved for up to an additive constant whose value is inconsequential in obtaining the solution for the flow. Furthermore, it can be confirmed by simple algebra that the coefficients $a_{j}$ decay exponentially as $j \rightarrow \pm \infty$, since the coefficients $b_{k, j}^{\prime}$ of the periodic boundary data decay exponentially as well. Thus, given the motions $U_{k}$ and $\Omega_{k}$ and the appropriate conformal mapping function $z(\zeta)$, one can explicitly form all the components to construct a solution of the form (10).

Some care must be taken when numerically truncating the series (10). When bodies are rather close in proximity, more terms of the series must be retained to provide an adequate representation of the potential since the conformal radius $\rho_{2}$ approaches 1 . In the examples provided here, the series is truncated to $N$ terms such that

$$
\frac{\left|a_{N}\right|^{2}}{\sum_{j=1}^{N-1}\left|a_{j}\right|^{2}}<0.01
$$

thus retaining $99 \%$ of the energy associated in the expansion. This typically leads to expansions with $N<50$ terms for separation distances $\epsilon / L \approx \mathcal{O}\left(10^{-2}\right)$, where $L$ is a pertinent length scale. Alternatively, a more elaborate truncation correction method can be used to combat the errors associated with representing the infinite series with a finite number of terms. Landweber and Shahshalan ${ }^{7}$ discuss a method where the "trapezoidal" remainder left from truncation is used to correct the truncated series. Without the correction, it is necessary to retain an additional $N / N_{\text {corrected }}=2.5$ terms to attain the same level of accuracy for gap distances of $\epsilon / L=0.01$ for the case of the impact of two circular cylinders; thus it is chosen to ignore the correction for simplicity, although at a higher computational expense.

\section{HYDRODYNAMIC COUPLING}

In this section, a method for determining the forces and moments and hence the coupled interactions of the two bodies is determined by employing the conservation of momentum and angular momentum.

\section{A. Hydrodynamic forces and moment}

The force on the body can be obtained via complex contour integrals around the objects by extension of the well known unsteady Blasius formula. ${ }^{21}$ Sedov $^{3}$ gives an expression for the complex force $F_{k}=f_{k, x}+i f_{k, y}$ and, in the absence of point singularities, it reduces to (with fluid density $\varrho_{f}$ )

$$
F_{k}=\overline{\frac{i \varrho_{f}}{2} \oint_{\partial D_{k}}\left(\frac{\mathrm{d} W}{\mathrm{~d} z}\right)^{2} \mathrm{~d} z}+i \varrho_{f} \frac{\mathrm{d}}{\mathrm{d} t}\left[\oint_{\partial D_{k}} z \frac{\mathrm{d} W}{\mathrm{~d} z} \mathrm{~d} z\right]+\varrho_{f} A_{k} \ddot{z}_{k},
$$

where $A_{k}$ and $z_{k}$ are the area and centroid position of the $k$ th object, respectively. It is seen that a "negative-mass" like term is introduced by the last term on the right hand side of Eq. (16). This may be deceptive since it is counterintuitive to the notion of added-mass, but the time derivative of the second term of Eq. (16) produces an additional "mass" term. In later examples, this term is found to counteract the negative- mass term and produce an overall positive added-mass for the system bodies.

Similarly, from Ref. 22, the torque applied by the fluid onto the body about its moving centroid in the laboratory frame is found to be

$$
\begin{aligned}
T_{k}= & \frac{\varrho_{f}}{2} \operatorname{Re}\left[2 \dot{\overline{z_{k}}} \oint_{\partial D_{k}}\left(z-z_{k}\right) \frac{\mathrm{d} W}{\mathrm{~d} z} \mathrm{~d} z-\oint_{\partial D_{k}}\left(z-z_{k}\right)\right. \\
& \left.\times\left(\frac{\mathrm{d} W}{\mathrm{~d} z}\right)^{2} \mathrm{~d} z+\frac{\mathrm{d}}{\mathrm{d} t} \oint_{\partial D_{k}}\left|z-z_{k}\right|^{2} \frac{\mathrm{d} W}{\mathrm{~d} z} \mathrm{~d} z\right]
\end{aligned}
$$

There are no further simplifications that can be made without prior knowledge of the form of the mapping function $z(\zeta)$. One can transform Eqs. (16) and (17) from integrals in the $z$-plane to integrals in the $\zeta$-plane. Thus, Eqs. (16) and (17) can be replaced, respectively, by

$$
\begin{aligned}
F_{k}= & \overline{i \varrho_{f}} \oint_{|\zeta|=\rho_{k}}\left(\frac{\mathrm{d} w}{\mathrm{~d} \zeta}\right)^{2}\left(\frac{d z}{d \zeta}\right)^{-1} \mathrm{~d} \zeta \\
& +i \varrho_{f} \frac{\mathrm{d}}{\mathrm{d} t}\left[\oint_{|\zeta|=\rho_{k}} z(\zeta) \frac{\mathrm{d} w}{\mathrm{~d} \zeta} \mathrm{d} \zeta\right]+\varrho_{f} A_{k} \ddot{z}_{k}
\end{aligned}
$$

and

$$
\begin{aligned}
T_{k}= & \frac{\varrho_{f}}{2} \operatorname{Re}\left\{2 \dot{\overline{z_{k}}} \oint_{|\zeta|=\rho_{k}}\left[z(\zeta)-z_{k}\right] \frac{\mathrm{d} w}{\mathrm{~d} \zeta} \mathrm{d} \zeta-\oint_{|\zeta|=\rho_{k}}\left[z(\zeta)-z_{k}\right]\right. \\
& \left.\times\left(\frac{\mathrm{d} w}{\mathrm{~d} \zeta}\right)^{2}\left(\frac{\mathrm{d} z}{\mathrm{~d} \zeta}\right)^{-1} \mathrm{~d} \zeta+\frac{\mathrm{d}}{\mathrm{d} t} \oint_{|\zeta|=\rho_{k}}\left|z(\zeta)-z_{k}\right|^{2} \frac{\mathrm{d} w}{\mathrm{~d} \zeta} \mathrm{d} \zeta\right\},
\end{aligned}
$$

where all integrals are now evaluated in the $\zeta$-plane. The integrals can be evaluated using residue theory as in Ref. 14, but due to the series form of $w(\zeta)$ and the geometry of the doubly connected domain, formulas (18) and (19) are best integrated numerically by parametrizing the curves $|\zeta|=\rho_{k}$ as $\zeta=\rho_{k} \exp (i \sigma)$ and integrating from 0 to $2 \pi$. This gives a numerical integration of a periodic function on a periodic domain and thus the integration can be carried out with spectral accuracy by employing a trapezoidal-rule quadrature.

It is also worthy to note that Eqs. (18) and (19) are extremely useful in the presence of discrete vortices as they remove the contour integration around the logarithmic singularities that are present in the standard formulation of the unsteady Blasius formula. ${ }^{22}$ Crowdy $^{12}$ has developed a novel method to add vortices to multiply connected domains and it can be readily applied to the current problem. 


\section{B. Coupled fluid-structure interactions}

The equations dictating the coupled fluid-structure motion are

$$
\begin{aligned}
& \left(\varrho_{b, k}-\varrho_{f}\right) A_{k} \ddot{z}_{k}=\overline{\frac{i \varrho_{f}}{2} \oint_{|\zeta|=\rho_{k}}\left(\frac{\mathrm{d} w}{\mathrm{~d} \zeta}\right)^{2}\left(\frac{\mathrm{d} z}{\mathrm{~d} \zeta}\right)^{-1} \mathrm{~d} \zeta} \\
& +i \varrho_{f} \frac{\mathrm{d}}{\mathrm{d} t}\left[\oint_{|\zeta|=\rho_{k}} z(\zeta) \frac{\mathrm{d} w}{\mathrm{~d} \zeta} \mathrm{d} \zeta\right]
\end{aligned}
$$

and

$$
\begin{aligned}
I_{k} \ddot{\theta}= & \frac{\varrho_{f}}{2} \operatorname{Re}\left\{2 \dot{\overline{z_{k}}} \oint_{|\zeta|=\rho_{k}}\left[z(\zeta)-z_{k}\right] \frac{\mathrm{d} w}{\mathrm{~d} \zeta} \mathrm{d} \zeta-\oint_{|\zeta|=\rho_{k}}\left[z(\zeta)-z_{k}\right]\right. \\
& \left.\times\left(\frac{\mathrm{d} w}{\mathrm{~d} \zeta}\right)^{2}\left(\frac{\mathrm{d} z}{\mathrm{~d} \zeta}\right)^{-1} \mathrm{~d} \zeta+\frac{\mathrm{d}}{\mathrm{d} t} \oint_{|\zeta|=\rho_{k}}\left|z(\zeta)-z_{k}\right|^{2} \frac{\mathrm{d} w}{\mathrm{~d} \zeta} \mathrm{d} \zeta\right\}
\end{aligned}
$$

with $\varrho_{b, k}$ being the density, $I_{k}$ being the moment of inertia of the $k$ th object, and $\theta_{k}$ being the orientation of body $k$ such that $\dot{\theta}_{k}=\omega$. One must also take care if numerically integrating the system. Since a time derivative given on the right hand side of Eqs. (20) and (21) gives rise to an added-mass type term, at each time step an iterative scheme must be implemented to ensure that the accelerations are consistent. Details of the are given in the Appendix.

\section{TWO INTERACTING CIRCULAR CYLINDERS}

Here, an example of two interacting circular cylinders is more closely investigated using the prescribed method. Due to the symmetry of the problem there, is no need to introduce rotations and torques on the bodies.

\section{A. Conformal map}

Two disks lie at $z_{1}(t)$ and $z_{2}(t)$ with radii $r_{1}=1$ and $r_{2}$ in the $z$-plane. The Möbius transformation that takes the annulus in the $\zeta$-plane $\left(\rho_{2}<|\zeta|<1\right)$ to the appropriate configuration in the $z$-plane is

$$
z=z_{1}+\exp (i \alpha) \frac{\zeta-\lambda}{\lambda \zeta-1}
$$

with

$$
\lambda=\frac{1+\left(\hat{x}_{2}^{2}-r_{2}^{2}\right)+\sqrt{\left(l_{1}^{2}-1\right)\left(l_{2}^{2}-1\right)}}{2 \hat{x}_{2}},
$$

$\alpha=\arg \left(z_{2}-z_{1}\right), \quad \hat{x}_{2}=\left|z_{2}-z_{1}\right|, \quad l_{1}=\hat{x}_{2}-r_{2}$, and $l_{2}=\hat{x}_{2}+r_{2}$ (see Ref. 20). The radius of the inner annulus, i.e., the conformal modulus, is found to be

$$
\rho_{2}=\frac{\left(\hat{x}_{2}^{2}-r_{2}^{2}\right)-1-\sqrt{\left(l_{1}^{2}-1\right)\left(l_{2}^{2}-1\right)}}{2 r_{2}} .
$$

The conformal map (22) can be easily inverted to give the inverse relationship that takes the physical plane to the mapped plane. We also note that in the special case where $r_{1}=r_{2}$, it can be shown that $\lambda^{2} \rho_{2}=1$.

\section{B. Complex potential solution}

Analytical expressions for the coefficients (14) can be obtained by expanding the boundary data via analytic continuation and integrating around the contour $|\zeta|=\rho_{k}$. Defining $B_{k}=\bar{U}_{k}\left(\lambda^{2}-1\right) \exp (i \alpha)$ and $C_{k}=\bar{U}_{k}\left[z_{1}+\lambda^{-1} \exp (i \alpha)\right]-U_{k}\left[\bar{z}_{1}\right.$ $+\lambda \exp (-i \alpha)]$, the boundary data can be expressed as

$$
\operatorname{Im}\left[\bar{U}_{1} z(\zeta)\right]=\frac{i \bar{B}_{1}}{2(\zeta-\lambda)}+\frac{i B_{1}}{2 \lambda(\lambda \zeta-1)}+\frac{i C_{1}}{2}, \quad \text { on }|\zeta|=1,
$$

$$
\begin{aligned}
\operatorname{Im}\left[\bar{U}_{2} z(\zeta)\right]= & \frac{i B_{2}}{2 \lambda(\lambda \zeta-1)}+\frac{i \rho_{2}^{2} \bar{B}_{2}}{2\left(\zeta-\lambda \rho_{2}^{2}\right)}-\frac{i C_{2}}{2}, \\
& \text { on }|\zeta|=\rho_{2} .
\end{aligned}
$$

Residue theory is used to integrate Eq. (14). Inserting Eqs. (23a) and (23b) into Eq. (14) gives residues at $\zeta=0,1 / \lambda$ and $\zeta=0, \lambda \rho_{2}^{2}$, which lie in their respective contours. Applying the residue theorem gives

$$
\begin{aligned}
& b_{1, j}=-\frac{i \bar{B}_{1}}{2 \lambda^{j+1}}, \\
& b_{2, j}=-\frac{i B_{2}}{2 \lambda^{1-j}},
\end{aligned}
$$

for $j \neq 0$ with the zero terms $b_{1,0}=-\operatorname{Im}\left\{U_{1}\left[\bar{z}_{1}+\lambda^{-1}\right.\right.$ $\times \exp (-i \alpha)]\}$ and $b_{2,0}=-\operatorname{Im}\left\{U_{2}\left[\bar{z}_{1}+\lambda \exp (-i \alpha)\right]\right\}$. Thus the coefficients in the Laurent expansion (15) are

$$
a_{j}=\frac{1}{\lambda^{j+1}\left(1-\rho_{2}^{2 j}\right)} \begin{cases}\bar{B}_{1}-B_{2}\left(\rho_{2} \lambda\right)^{2 j}, & j>0, \\ \bar{B}_{2}-B_{1} \lambda^{2 j}, & j<0 .\end{cases}
$$

The necessary compatibility condition sets the constant $d_{2}=\operatorname{Im}\left[\left(U_{2}-U_{1}\right) \bar{z}_{1}+\left(U_{2} \lambda-U_{1} \lambda^{-1}\right) \exp (-i \alpha)\right]$.

\section{Forces induced from inline impact of two circular cylinders}

To validate the method, the inline impact of two cylinders is investigated and the results are compared to Wang, ${ }_{7}^{8}$ Bampalas and Graham, ${ }^{14}$ and Landweber and Shahshahan. ${ }^{7}$ For simplicity, the cylinder radii are taken to be $r_{1}=r_{2}=1$ $(D=2)$. The integrals for the force given by Eq. (16) are integrated numerically using a trapezoidal-rule quadrature. Since motions are prescribed, a simple first order finite difference scheme is used to approximate the time derivative to $\mathcal{O}\left(10^{-8}\right)$.

First we investigate the inline motion of a cylinder $(k=2)$ toward a stationary cylinder $(k=1)$. Cylinder 2 moves at a constant velocity $U_{2}$. Streamlines for such a motion are shown in Fig. 2. In Fig. 3, the force coefficient in the $x$-direction $C_{x}=2 f_{x} /\left(\varrho_{f} U_{2}^{2} D\right)$ for both the moving and stationary cylinders are plotted versus nondimensional time $t^{*}=U_{2}\left(t-t_{0}\right) / D$. The two cylinders impact at $t^{*}=0$. In addition to results from the current formulation, data from the papers of Bampalas and Graham, ${ }^{14}$ Landweber and Shahshahan, ${ }^{7}$ and Wang ${ }^{8}$ are also plotted for comparison. It 


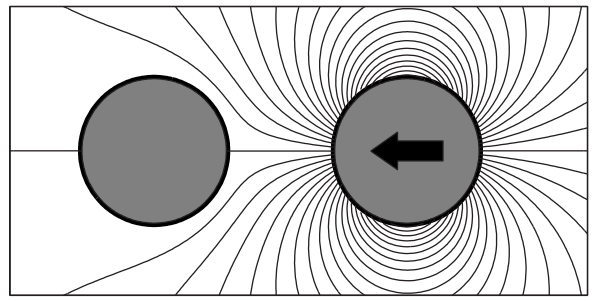

FIG. 2. Instantaneous streamlines due to a cylinder approaching a fixed cylinder from the right at constant velocity.

is useful to note that unlike the current method, Bampalas and Graham ${ }^{14}$ and Wang ${ }^{8}$ use a form of the Blasius equation much like the formulas given in Milne-Thomson ${ }^{21}$ but with assumptions that allowed the order of the spatial integration and time derivative of $\Phi$ to be interchanged. On the other hand, Landweber and Shahshahan ${ }^{7}$ used a Lagrangian mechanics perspective to derive the force on the cylinders as outlined in Lamb. ${ }^{23}$ Using the current method, the agreement to Bampalas and Graham ${ }^{14}$ and Landweber and Shahshahan ${ }^{7}$ is excellent for both the moving and stationary cylinders. As the cylinders approach each other, the forces become unbounded as expected. The results from Wang ${ }^{8}$ for the stationary cylinder agree well, but the results for the moving cylinder are offset due to the incorrect treatment of the Blasius force equation. This difference is accounted for by the omission of a convective term $U_{2} \partial \Phi / \partial x$ in the standard Blasius equation as discussed in Ref. 14. The Sedov formula presented in Eq. (16) accounts for such a term in its derivation.

Additionally, a case where two cylinders move toward each other at a constant velocity $U_{1}=U_{2}=U$ is compared. Streamlines for such a case are given in Fig. 4. The magnitude of the force coefficient in the $x$-direction is plotted with the same nondimensional variables as given above. Figure 5 shows the results for this configuration in comparison to the data from Bampalas and Graham ${ }^{14}$ and Wang. ${ }^{8}$ Again, the

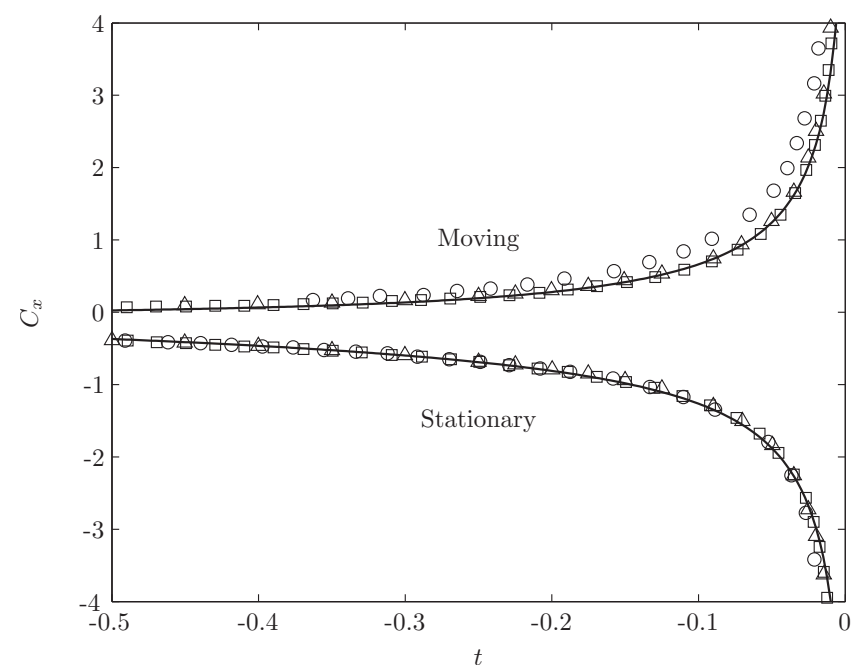

FIG. 3. Force coefficient $C_{x}$ when a cylinder moves toward a stationary cylinder as depicted in Fig. 2. The two cylinders impact at $t^{*}=0$. Present method; $\square$, Bampalas and Graham (Ref. 14); $\bigcirc$, Wang (Ref. 8); and $\triangle$, Landweber and Shahshahan (Ref. 7).

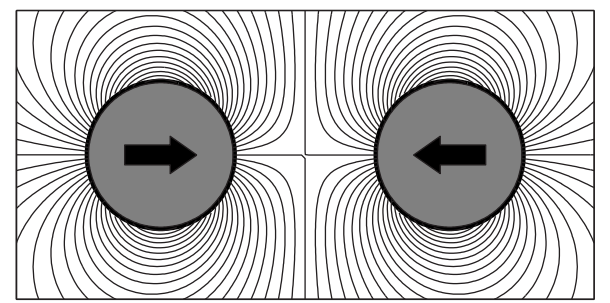

FIG. 4. Instantaneous streamlines due to two cylinders approaching each other inline at constant velocity.

agreement between Bampalas and Graham ${ }^{14}$ is nearly exact, while the results from $\mathrm{Wang}^{8}$ are slightly offset due to the mistaken omission of the convective term.

As previously discussed, when the bodies become closer in proximity, more terms in the expansion (25) are retained for an accurate representation in the truncated series. Given in Fig. 6 is the convergence of the coefficients $a_{j}$ when the cylinders are separated by a distance $\epsilon / D=0.01$ to highlight the spectral convergence of the Laurent coefficients in this case. The square of the magnitude of the $j$ th term is machine precision small at $j \approx 150$. For the investigations hereafter, a minimum separation distance of $\epsilon / D=0.01$ is maintained.

\section{Coupled dynamics of two circular cylinders}

For coupled cylinder interactions we employ Eq. (20). The equations are integrated using a variable-time step explicit Runge-Kutta solver in MATLAB (i.e., the ODE45 package) with strict relative and absolute tolerances on the error in the solution $\left(10^{-6}\right.$ and $10^{-8}$, respectively). Here we study two different types of cases. First, we investigate the influence that a cylinder with prescribed motion has on a cylinder that is allowed to freely interact with the flow (forced-free interaction). Second, we study the case where both cylinders are given an initial condition and allowed to interact freely with one another (free-free interaction). In what follows it is

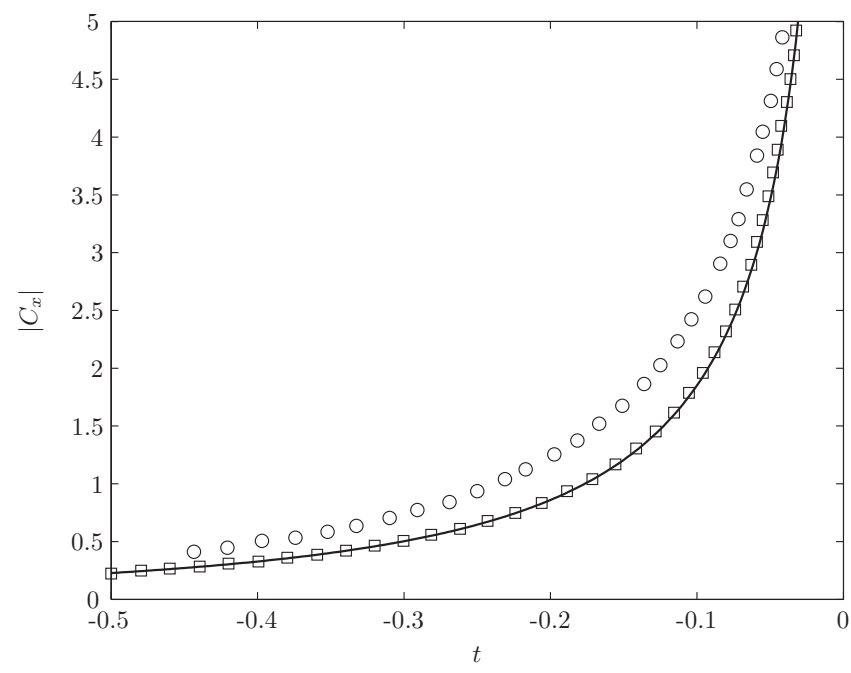

FIG. 5. Magnitude of the force coefficient $\left|C_{x}\right|$ when two cylinders move toward each other at constant velocity as depicted in Fig. 4. The two cylinders impact at $t^{*}=0$. — , Present method; $\square$, Bampalas and Graham (Ref. 14); and $\bigcirc$, Wang (Ref. 8). 


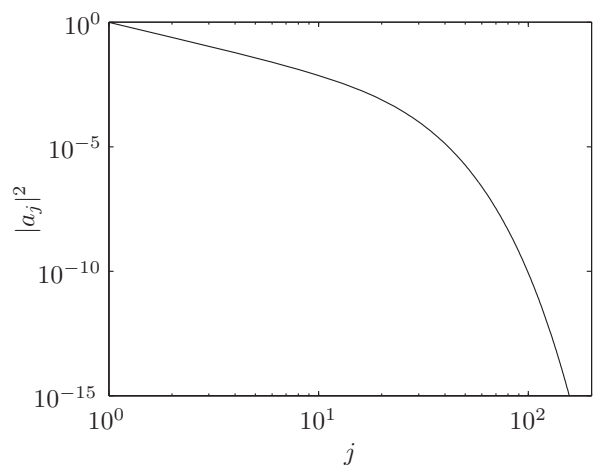

FIG. 6. Convergence of $\left|a_{j}\right|^{2}$ vs $j$. For the negative terms, the scaled coefficients $a_{-j} \rho_{2}^{-j}$ reveal the same trend.

assumed that each body is of equal radius $r_{1}=r_{2}$, and neutrally buoyant $\varrho_{b, k}=\varrho_{f}$.

\section{Forced-free interaction applied to hydrodynamic drafting}

In this section, we prescribe the motion of the first body and allow the second body to move freely in the flow. One particular area of application is to characterize how bodies forward of another body affect the hydrodynamic performance of the aft body. Here we look to investigate the benefits that the aft body receives in "riding" behind the forward cylinder that has a prescribed motion, primarily to determine good drafting regions. We first initialize both cylinders to have unit velocity in the $x$-direction, i.e., $U_{k}=1$. It is assumed that the forward body travels at a uniform speed $U_{1}=1$ (which means there must be an external force on it). No external force is applied to the second body so it moves in response to the resultant fluid forces. A few examples are given in Fig. 7. If the aft cylinder sits in a narrow band directly behind the forward cylinder, its velocity is decreased. On the other hand, the aft cylinder is capable of maintaining or overtaking the forward cylinder with no added force if it sits more toward the forward cylinder's side. In one case, it is seen that the aft cylinder is ejected in the $y$-direction due to the large side force generated from the motion of the forward cylinder.

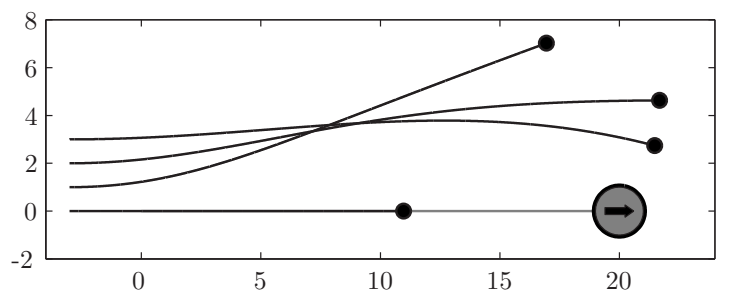

FIG. 7. Several example paths of an aft cylinder following the forced motion of a forward cylinder. Both cylinders are initially moving to the right with unit speed. Black lines denote the paths of the passive cylinder, - denotes the centroid location at $t=20$, and the gray line denotes the path of the forward cylinder. The forward cylinder is forced at a constant velocity starting from $z_{1}(0)=0$. The initial locations for the aft cylinder are $z_{2}(0)=-3,-3+i,-3+2 i$, and $-3+3 i$.

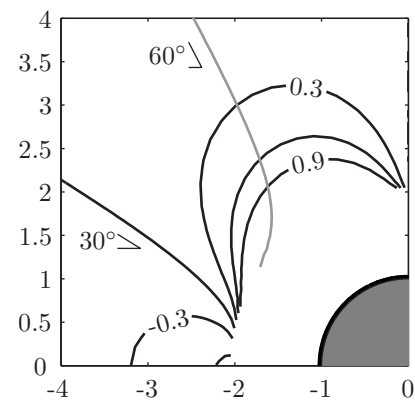

(a)

FIG. 8. Contour plots for the force on an aft cylinder drafting behind another cylinder of equal radius. Both the lead cylinder and trailing cylinder move to the right with $U_{1}=1$. (a) Thrust force acquired by the aft cylinder based on the location of its center. The gray curve indicates the location where the side force on the aft body is exactly zero. (b) Side force on the aft cylinder. Contours on all plots are separated by an increment of 0.3 .

To further elucidate the effect of drafting for the aft body, we look at the forces that the aft body experiences due to the presence of the forward body. In a perfect fluid, a single cylinder in an unbounded flow travels with no drag and thus does not require any energy input to maintain its velocity. Thus we look for the regions in a force contour map where the aft cylinder receives an additional thrust due to the motion of the forward body. This extra force assists the aft body to overcome the drag it is to experience if in a viscous fluid. Plotted in Figs. 8(a) and 8(b) are the thrust force and side force experienced by the aft body, respectively. Although it may seem beneficial to draft directly behind the lead cylinder since fluid rushes to fill the forward mass, doing so actually hinders the following body substantially. The aft body receives an induced hydrodynamic drag due to the high-pressure region created between the bodies. Thus, as seen in a particular path in Fig. 7 where the aft body is initialized directly behind the forward cylinder, the separation between the two increases as they move toward the right. Alternatively, if the aft cylinder sits in an area slightly behind the forward cylinder's midline but above the $30^{\circ}$ angle line [see Fig. 8(a)], the forward cylinder experiences a thrust force allowing the aft cylinder to be propelled beyond the forced cylinder as seen in a few of the example path lines given in Fig. 7. This force increases as the distance between the cylinders is reduced and reaches a finite value in the limit that the separation is the sum of their radii. In addition to the thrust force, the aft body can experience a significant side force as seen in Fig. 8(b). In many applications, such as the drafting of neonate dolphins behind their mothers, the side force can be a substantial limiting factor on the effectiveness of drafting. In Fig. 8(a), the line of the zero side force is shown in gray to give a sense of the location where the aft cylinder feels the zero side force, thus allowing the aft cylinder to stay near the body without being pushed away or colliding with the forward cylinder. This line indicates that the preferred location for drafting is roughly inclined at an angle of $60^{\circ}$ from the trailing edge of the forward cylinder. 


\section{Free-free interaction applied to the collision of two cylinders}

We now investigate the dynamics of two circular bodies that are allowed to interact with one another given a specific set of initial conditions. As a specific example, the collision of two circular cylinders is studied to illustrate the effect of the fluid on the resultant motion of the cylinders before, after, and at the time of impact.

Consider the assumed collision of two cylinders. When two circular cylinders collide, the momentum along the line of contact is transferred between the two bodies. For a perfectly elastic collision, this transfer of momentum between the two bodies must satisfy both the conservation of total momentum and the conservation of energy. We seek to enforce this when the two bodies collide and to do so, expressions for the total momentum and energy are derived and used to determine the resultant velocities after impact. The momentum of both the bodies and the fluid is written as an integral over the entire plane $A$,

$$
\mathbf{P}=\int_{A} \varrho \mathbf{u} \mathrm{d} A=m\left(\mathbf{U}_{1}+\mathbf{U}_{2}\right)+\varrho_{f} \int_{A_{f}} \nabla \Phi \mathrm{d} A
$$

where explicit integration over the two bodies leaves us with the standard expression for momentum of two rigid bodies, each with mass $m$, and an expression for the fluid momentum as an integral over the region occupied by the fluid $A_{f}$. By applying the divergence theorem to the area integral over the fluid region, Eq. (26) can be rewritten as

$$
\mathbf{P}=m\left(\mathbf{U}_{1}+\mathbf{U}_{2}\right)-\oint_{C_{1}+C_{2}} \Phi \hat{\mathbf{n}} \mathrm{d} s .
$$

Now, we take the vector equation (27) and transform it to the complex notation $\mathbf{P} \mapsto P=P_{x}+i P_{y}$ by noting that the complex version of the normal can be represented with $\hat{\mathbf{n}} \mapsto$ $-i(\mathrm{~d} z / \mathrm{d} s)$ and recalling that $\Phi=\operatorname{Re}[W]$. Thus,

$$
P=m\left(U_{1}+U_{2}\right)+\rho_{f} \oint_{C_{1}+C_{2}} \operatorname{Re}[W] \mathrm{d} z .
$$

Similarly, the expression for kinetic energy is written as

$$
\begin{aligned}
E & =\frac{1}{2} \int_{A} \varrho(\mathbf{u} \cdot \mathbf{u}) \mathrm{d} A \\
& =\frac{m}{2}\left(\left|\mathbf{U}_{1}\right|^{2}+\left|\mathbf{U}_{2}\right|^{2}\right)+\frac{\underline{\varrho}_{f}}{2} \int_{A_{f}}(\nabla \Phi \cdot \nabla \Phi) \mathrm{d} A,
\end{aligned}
$$

where the last integral is the kinetic energy of the fluid alone. The area integral for the kinetic energy of the fluid in Eq. (29) can be transformed to a contour integral by applying Green's theorem and noting that $\nabla^{2} \Phi=0$. Thus

$$
\int_{A_{f}}(\nabla \Phi \cdot \nabla \Phi) \mathrm{d} A=-\int_{C_{1}+C_{2}} \Phi \frac{\partial \Phi}{\partial n} \mathrm{~d} s+\int_{C_{\infty}} \Phi \frac{\partial \Phi}{\partial n} \mathrm{~d} s
$$

where $C_{\infty}$ is a contour that extends to infinity. The last integral in Eq. (30) is exactly zero due to the continuity equation and the requirement that $\Phi$ must be a constant at infinity;

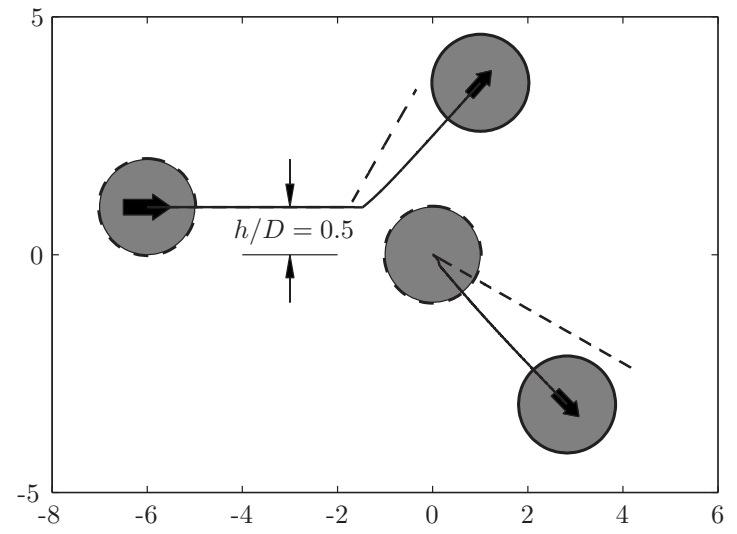

FIG. 9. Paths of two cylinders in a collision event. The dashed filled cylinders indicate the initial locations and velocities $\left[U_{1}(0)=1, U_{2}(0)=0\right.$, $z_{1}(0)=-6+i$, and $\left.z_{2}(0)=0\right]$, while the solid filled cylinders indicate the resultant locations and velocities at $t=10$. In addition to the paths taken by the cylinders in a two-dimensional inviscid fluid (solid line), the paths of a perfect collision in the absence of the fluid are also plotted (dashed line) for comparison. Arrows scale with the magnitude of velocity.

thus, when placed in complex form, Eq. (29) reads

$$
\begin{aligned}
E= & \frac{m}{2}\left(\left|U_{1}\right|^{2}+\left|U_{2}\right|^{2}\right)-\frac{\varrho_{f}}{2} \oint_{C_{1}} \operatorname{Re}[W] \operatorname{Re}\left[-i \bar{U}_{1} \mathrm{~d} z\right] \\
& -\frac{\varrho_{f}}{2} \oint_{C_{2}} \operatorname{Re}[W] \operatorname{Re}\left[-i \bar{U}_{2} \mathrm{~d} z\right] .
\end{aligned}
$$

Equations (28) and (31) must be conserved following a collision event.

To model a collision, a threshold value $\epsilon$ is chosen such that when separation between the cylinders is below this value, a collision takes place. This threshold value is selected to be $\epsilon=0.01 D$ for all the cases presented here. If a collision event occurs at $t=t_{c}$ while the equation of motion (20) is integrated, the preimpact velocities are decomposed into two components: a component that is along the line of collision $\left[U_{k, n}\left(t_{c}^{-}\right)\right]$and a component that is tangent to the line of collision $\left[U_{k, t}\left(t_{c}^{-}\right)\right]$. The change in resultant velocity after a collision must only take place in the direction along the line of collision while conserving both the total momentum and energy of the system. For example, in the absence of the fluid, closed-form expressions for the post-impact normal velocities of the two equal mass cylinders are easily found to be $U_{1, n}\left(t_{c}^{+}\right)=U_{2, n}\left(t_{c}^{-}\right)$and $U_{2, n}\left(t_{c}^{+}\right)=U_{1, n}\left(t_{c}^{-}\right)$, while the tangential component remains the same. With the presence of the fluid, we resort to numerically determining the post-impact normal velocities $U_{k, n}\left(t_{c}^{+}\right)$by ensuring that the total normal momentum $\left[P_{n}\left(t_{c}^{+}\right)=P_{n}\left(t_{c}^{-}\right)\right]$and the total energy $\left[E\left(t_{c}^{+}\right)=E\left(t_{c}^{-}\right)\right]$are conserved.

As an example, an oblique collision event is plotted in Fig. 9. Here, a cylinder starting from the left is initialized with $U_{1}(0)=1$ and $z_{1}(0)=-6+i$, and allowed to interact with the second cylinder at rest with $U_{2}(0)=0$ and position $z_{2}(0)=0$. In the presence of the fluid, the cylinders collide at $t=4.45$. The paths for such a situation are given in Fig. 9 as black lines. For comparison, the paths for the case where the 

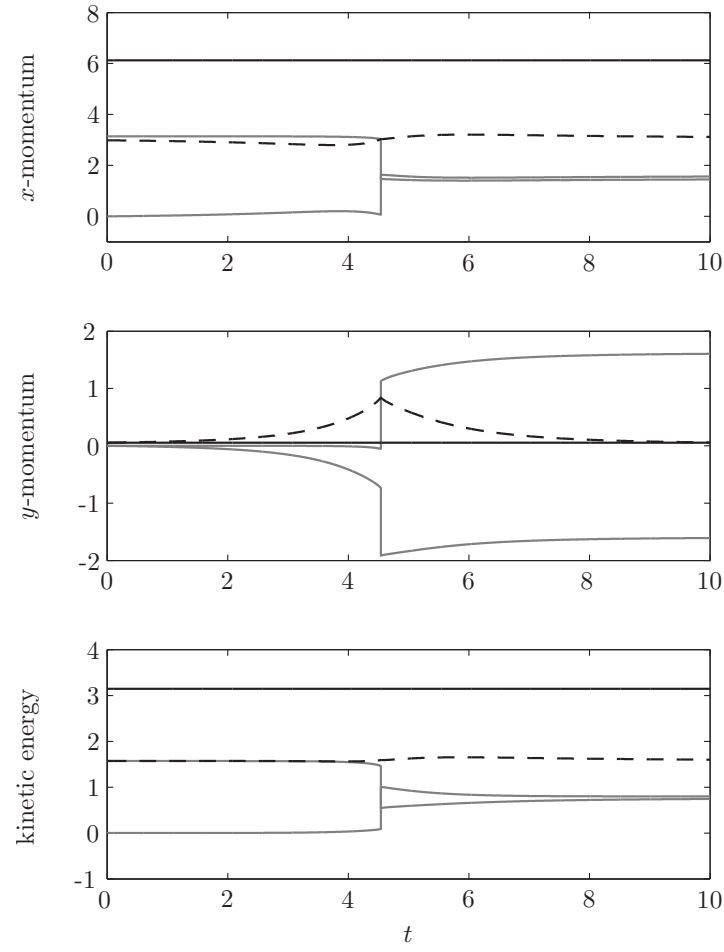

FIG. 10. The momentum $P$ and kinetic energy $E$ of the fluid (dashed line), each body (gray line), and total system (black line) are given for the oblique collision event presented in Fig. 9. The collision occurs at $t=4.45$.

fluid is absent are also plotted as dashed lines. One can see a distinct difference between the two paths due to the presence of the fluid.

To validate this type of behavior, the evolution of momentum and energy for the fluid, each body, and the entire system are plotted in Fig. 10. It is easily seen that the total momentum and energy of the system is conserved in addition to providing more justification for the use of Eq. (20) for modeling fluid-structure interaction in potential flow. In all instances presented here, the normalized variations in total momentum and kinetic energy are maintained at $\mathcal{O}\left(10^{-3}\right)$ or less. It is interesting to observe that the fluid momentum and energy is continuous at the instant of collision (although nonsmooth). The lack of the jump in the fluid momentum actually indicates that at the time of impact, the transfer of momentum between the two bodies is conserved, that is, even though we enforce the requirement that the total momentum of the entire fluid-body system to be conserved, the collision itself degenerates into satisfying only the momentum transfer between the two bodies. In essence, at the time of collision, the fluid can be neglected, although the fluid plays an important role in deflecting and buffering the two bodies prior to collision, thus drastically affecting the resulting trajectories of both the impinging cylinder and the target cylinder by changing the line of impact.

Furthermore, it is also important to distinguish the difference between the fully coupled fluid-structure interaction and the prescribed motion case. As observed in Sec. IV C, the prescribed collision of two cylinders creates an unbounded force approaching impact. Intuitively, one thinks this suggests that a collision can never occur because the
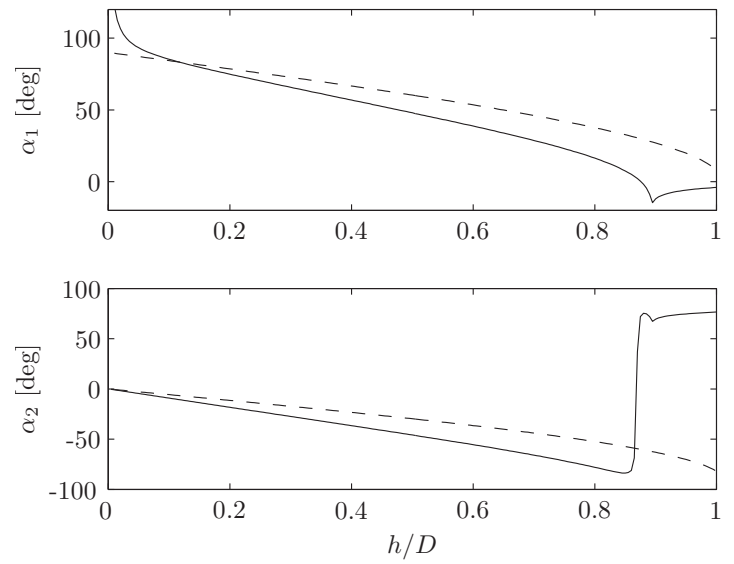

FIG. 11. Angles $\alpha_{1}$ and $\alpha_{2}$ that the cylinders are deflected vs $h / D$ after an oblique collision with the second cylinder initially at rest. With fluid present (solid line) and without the fluid present (dashed line).

forces become increasingly large as the cylinders approach. The key contrast in the fluid-structure interaction (FSI) case is that the bodies undergo deceleration as well, and this in turn affects the forces on the body. Obviously, if one thinks of the notion forces in this FSI case, they are counteracted by the inertia of the bodies $m \dot{U}_{k}$ and remain bounded up until the moment of the modeled impact as seen in Fig. 10. Thus it is hard to conclude from a prescribed interaction that there are any convincing trends for the fluid-structure interaction of multiple bodies.

Plotted in Fig. 11 are $\alpha_{1}=\arg \left[U_{1}(\infty)\right]$ and $\alpha_{2}$ $=\arg \left[U_{2}(\infty)\right]$, i.e., the angles that the postcollision velocity of the impinging and target bodies make with the horizontal versus an impinging obliqueness measure $h / D$ (see Fig. 9 for definition). As Fig. 11 demonstrates, the difference between a collision without and with the fluid is that the impact is shallower due to the fluid slightly moving the target cylinder away from the impact. In the region $0.845<h / D<0.895$, the collision is so shallow that the initially stationary cylinder actually begins to move to fill the void of fluid following the impinging cylinder, albeit at a very small resultant velocity, thus the tendency for the second cylinder to have a positive $\alpha_{2}$. When $h / D>0.895$, the incoming cylinder narrowly misses the target cylinder whereas when the fluid is absent, the cylinders actually collide. In this sense, the fluid acts as a buffer to avoid a collision. However, the fluid facilitates a transfer of momentum between the two cylinders through the low pressure region existing between the bodies and thus, without any direct contact between the cylinders, the incoming cylinder is deflected downward quite substantially. In addition, the initially stationary cylinder gains a substantial amount of momentum, thus propelling itself primarily in the $y$-direction. This process is illustrated in Fig. 12 and demonstrates a substantial hydrodynamic drift for the target cylinder in the direction transverse to the dominant motion in the fluid. For this specific case where $U_{1}(0)=1, U_{2}(0)=0$, and $z_{1}(0)=-6+1.8 i$, the resultant velocities of the bodies are $U_{1}(\infty)=0.954-0.205 i$ and $U_{2}(\infty)=0.030+0.216 i$. As $h / D$ increases, the influence that the fluid has on the interaction of the two bodies begins to degrade rapidly. 


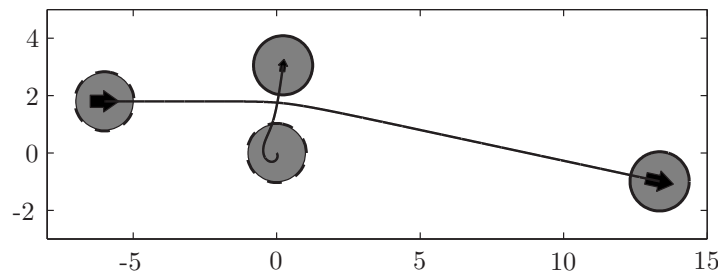

FIG. 12. Cylinder paths for a near-collision event. The initial conditions are $U_{1}(0)=1, U_{2}(0)=0, z_{1}(0)=-6+1.8 i$, and $z_{2}(0)=0$.

\section{A PAIR OF FLAPPING PARALLEL PLATES}

An additional example is given here to highlight the use of the method for more complex body shapes. Here, a brief account of applying the method to a pair of flapping plates is given.

\section{A. Conformal map}

Consider two parallel flat plates defined by the two angles $\chi$ and $\beta$, separation distance $l$, plate chord length for each plate $c$, and midpoint location $z_{0}$, as depicted in Fig. 13 . The conformal map taking the annulus to the two parallel plates is given by

$$
\begin{aligned}
z= & \tau\left[K\left(\zeta / \sqrt{\rho_{2}}, \rho_{2}\right)-\exp (2 i \nu) K\left(\zeta \sqrt{\rho_{2}}, \rho_{2}\right)-K\left(-1, \rho_{2}\right)\right. \\
& \left.-\exp (2 i \nu) K\left(-\rho_{2}\right)\right]+z_{0},
\end{aligned}
$$

where

$$
K\left(\zeta, \rho_{2}\right)=1-2 \zeta \frac{P^{\prime}\left(\zeta, \rho_{2}\right)}{P\left(\zeta, \rho_{2}\right)}
$$

and

$$
P\left(\zeta, \rho_{2}\right)=(1-\zeta) \prod_{j=1}^{\infty}\left(1-\rho_{2}^{2 j} \zeta\right)\left(1-\rho_{2}^{2 j} \zeta^{-1}\right)
$$

is the Schottky-Klein prime function for the annulus domain. ${ }^{24}$ The parameters $\tau, \rho_{2}$, and $\nu$ in Eq. (32) are all functions of time and can be determined implicitly (e.g., using Newton's method) by enforcing the geometric conditions

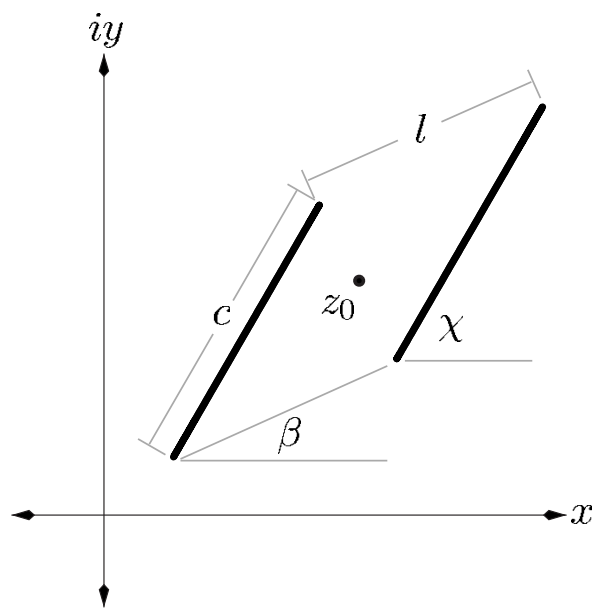

FIG. 13. Geometric quantities defining the configuration of two parallel plates in $z$-plane.

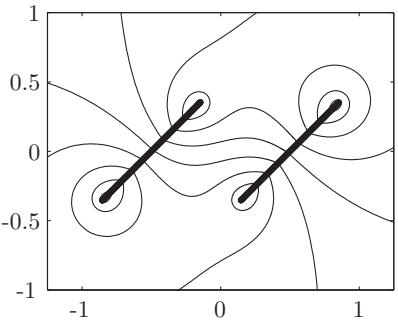

(a)

(b)

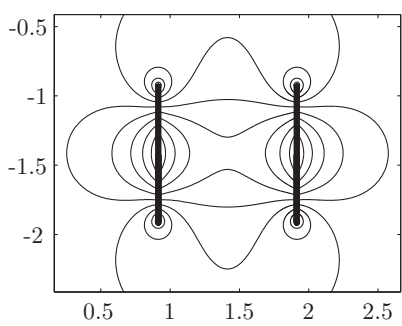

FIG. 14. Instantaneous streamlines for the flapping motion of two parallel plates with motion prescribed by Eq. (33). (a) $t=0$. (b) $t=\pi / 2$.

on $\beta, \chi, c$, and $l$ defining Fig. 13. Note that we can conveniently set $\theta_{k}=\chi$.

\section{B. Sinusoidal flapping and pitching}

Here, the configuration is restricted to $\beta=0$ and $c=l=1$ such that the plates are always stationed at the same $y$-location. A flappinglike wing motion is chosen such that the remaining geometric parameters are prescribed as follows:

$$
\begin{aligned}
& z_{0}=\exp \left(-\frac{i \pi}{4}\right) \sin (t), \\
& \chi=\frac{\pi}{2}\left[1-\frac{1}{2} \cos (t)\right] .
\end{aligned}
$$

The prescribed motion gives the downstroke a large angle of attack while the upstroke maintains a relatively small angle of attack (relative to the motion of the plates). Although idealized, the motion can be seen as a model of dragonfly wings beating where the forewings and hindwings are in phase.

The solution for the potential is given by Eq. (10) with the coefficients (15) determined numerically by the method discussed in Sec. II B. Figure 14 gives the streamlines during two instances of the flapping motion prescribed by Eq. (33). Figure 14(a) shows the streamlines at $t=0$ where the angle of attack is $90^{\circ}$ and $\Omega_{1}(0)=\Omega_{2}(0)=\dot{\chi}(0)=0$. Alternatively, Fig. 14(b) depicts the streamlines at $t=\pi / 2$ when the plates are experiencing pitch reversal for the upstroke. Here, the angular velocity is at a maximum $\dot{\chi}(t)(\pi / 2)=\pi / 4$, while there is no translational velocity.

Care must be taken to integrate Eqs. (18) and (19) since the mappings include four zeros on the annuli representing the four sharp edges in the physical plane. This in turn introduces four poles lying on the contours when attempting to integrate Eqs. (18) and (19). To make progress, the integration contour is stretched slightly into the fluid domain avoiding the singularities. The resulting force and moment coefficients acting about the plate centroids $z_{k}$, in addition to the sum of each component (e.g., $C_{x}=C_{1, x}+C_{2, x}$ ) for a single cycle, are given in Fig. 15. A few observations can be made. Contrary to the potential flow around a single plate in the absence of circulation, each plate experiences a force and moment and their sum is not simply zero. A net force and moment about $z_{0}$ may act on the entire system at any given 

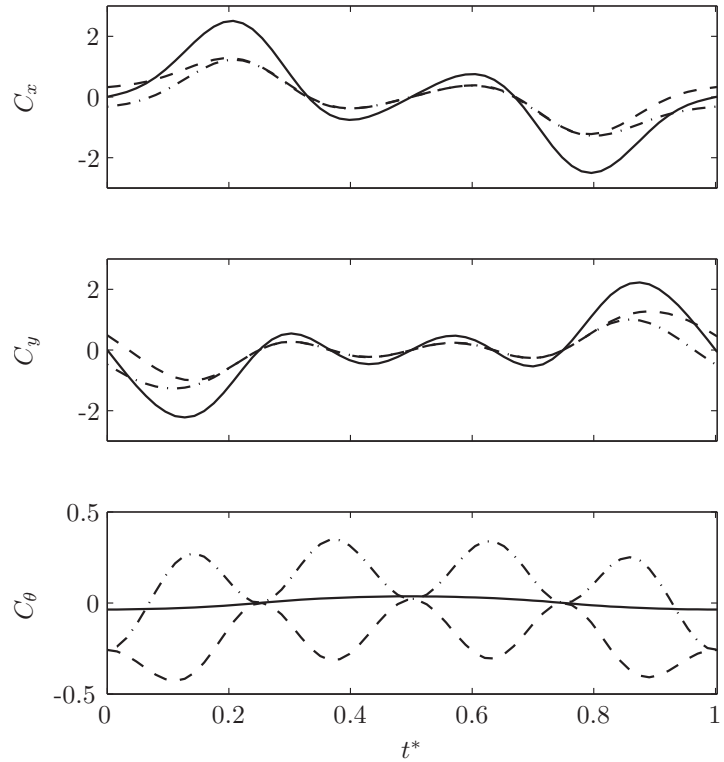

FIG. 15. Individual and total force and moment coefficients vs nondimensional time $t^{*}=t /(2 \pi)$ of two plates undergoing motion prescribed by Eq. (33) for one cycle. Lines indicate the sum of $k=1,2$ (solid line); $k=1$ (dashed line); and $k=2$ (dashed-dotted line). For $C_{\theta}$, the solid line represents the sum of the total torque about $z_{0}$.

instant in time, although the moment in this case is especially small. In addition, it appears that pitching in the positive $\chi$-direction during pitch reversal affects the lift force substantially and actually decreases lift in pure potential flow. Furthermore, for this sinusoidal motion, the net lift and torque $\int\left(C_{x}, C_{y}, C_{\theta}\right) \mathrm{d} t$ over one period is exactly zero. This suggests that although there are net forces and torques on the system at various instants in time, the sinusoidal motion restricts the net force or torque over one cycle to be zero.

\section{CONCLUSION}

A general method to solve for the complex potential of two bodies translating and rotating in a perfect fluid has been derived. The formulation can be used to form the complex potential given the conformal map of an arbitrary doubly connected domain to the annulus domain. Having solved for the complex potential, generalized forms of the Blasius formulas from Sedov can be used to calculate the forces and moments on both bodies by numerically evaluating contour integrals along each body in the annulus domain. The forces and moments can additionally be used to dynamically couple the two bodies to analyze their fluid-structure interaction.

This formulation can be used in various applications to study the dynamics of bodies that constitute a doubly connected domain with relative ease (e.g., Weis-Fogh mechanism, ground effect, dragonfly aerodynamics, etc.). One specific case considered here is the problem of two interacting cylinders in a perfect fluid. Three subcases are studied. First, the motion of the cylinders is prescribed and the forces experienced by both cylinders are calculated to provide validation for this method. It is demonstrated that when one cylinder is forced to collide with a stationary cylinder, the forces experienced by both cylinders are in excellent agreement with previous published data. In addition, when both cylin- ders are prescribed to impact each other at equal and opposite velocities, the agreement with previous data is also nearly exact.

In the second subcase, the motion of one of the cylinders is prescribed and the second cylinder moves only subject to the fluid forces it experiences. The analysis of the forced-free interaction is applied in the context to the benefits of hydrodynamic drafting. It is shown, contrary to intuition, that drafting directly behind a second body leads to substantial increased induced drag for neutrally buoyant cylinders. When the body sits in a particular zone, the body is capable of receiving additional thrust to aid its ability to overcome an unmodeled viscous drag while experiencing very little adverse side force. For example, this coupling may help give more insight on why neonate dolphins, among other aquatic animals, follow obliquely as opposed to directly behind their mothers, although we have neglected to model the effects of a possible separated flow (for example, see Ref. 25).

In the third subcase, two cylinders are allowed to dynamically interact with one another through their mutual induced forces specifically in oblique collision and nearcollision events. It is seen that the fluid provides a buffering effect prior to collision by attempting to push the colliding cylinders away from each other, but not necessarily preventing collision. In some instances with the presence of the fluid, the cylinders are capable of narrowly avoiding a collision, whereas in the absence of fluid, the two cylinders experience a collision event. In addition to possibly avoiding the collision, a substantial drift velocity in the direction normal to the motion of impinging body can be obtained.

Lastly, a brief example of a pair of flapping parallel plates is given to highlight the application of the proposed method in a more complicated domain. A dual wing configuration is further investigated in the context of flapping flight. It is found that each body is capable of sustaining a force or moment at any given instant of time, but under sinusoidal motion, the net force and torque about the system centroid averaged over a cycle are exactly zero. Though it is hard to draw any definitive conclusions without the inclusion of separated vortices in this high angle of attack application, it is clear that the sinusoidal pitching gives a hydrodynamic disadvantage in terms of producing net lift.

We would like to note that this two-dimensional study is highly idealized and does not capture many important effects of viscous, three-dimensional flows. Nevertheless, it is sensible to investigate this idealized system because it calls attention to several dynamical features that have remained unexplored. Even in cases where bodies exhibit substantial three-dimensional effects, the method presented here can lead to important generalizations in an idealized configuration to give some intuition for these fluid-structure interactions.

\section{ACKNOWLEDGMENTS}

A.A.T. wishes to acknowledge the discussions with P. K. Newton and E. Kanso that helped lead to some of the results given in this paper. This work is supported by the Air Force Office of Scientific Research, Award No. FA9550-05-1-0411. 


\section{APPENDIX: INTEGRATION IN TIME}

Care must be taken when numerically integrating the coupled FSI problems presented in Eqs. (20) and (21). For example, in Eq. (20), we cast the equations in the following form:

$$
\begin{gathered}
\frac{\mathrm{d} P_{k}}{\mathrm{~d} t}=G_{k}, \\
\frac{\mathrm{d} z_{k}}{\mathrm{~d} t}=U_{k},
\end{gathered}
$$

where

$$
\begin{aligned}
P_{k} & =\left(\varrho_{b}-\varrho_{f}\right) A_{k} U_{k}-i \varrho_{f} \oint_{|\zeta|=\rho_{k}} z(\zeta) \frac{\mathrm{d} w}{\mathrm{~d} \zeta} \mathrm{d} \zeta, \\
G_{k} & =\overline{\frac{i \varrho_{f}}{2} \oint_{|\zeta|=\rho_{k}}\left(\frac{\mathrm{d} w}{\mathrm{~d} \zeta}\right)^{2}\left(\frac{\mathrm{d} z}{\mathrm{~d} \zeta}\right)^{-1} \mathrm{~d} \zeta .}
\end{aligned}
$$

The integrals represented in Eq. (A2b) are implicit functions of the physical variables $z_{1}(t), z_{2}(t), U_{1}(t)$, and $U_{2}(t)$. One can integrate Eq. (A1) with any adequate time-integrator but it must be ensured that the term Eq. (A2a) is satisfied. To do so, one must solve Eq. (A2a) for a suitable $U_{k}$, e.g., via Newton iterations, for the use in Eq. (A2b) and subsequently in Eq. (A1).

${ }^{1}$ M. Lighthill, "Weis-Fogh mechanism of lift generation," J. Fluid Mech. 60, 1 (1973).

${ }^{2}$ Z. J. Wang and D. Russell, "Effect of forewing and hindwing interactions on aerodynamic forces and power in hovering dragonfly flight," Phys. Rev. Lett. 99, 148101 (2007).

${ }^{3}$ L. I. Sedov, Two-Dimensional Problems in Hydrodynamics and Aerodynamics (Wiley, New York, 1965).

${ }^{4}$ S. B. Furber and J. E. Ffowcswilliams, "Is the Weis-Fogh principle exploitable in turbomachinery," J. Fluid Mech. 94, 519 (1979).

${ }^{5}$ S. Nair and E. Kanso, "Hydrodynamically coupled rigid bodies," J. Fluid Mech. 592, 393 (2007).
${ }^{6}$ M. Lagally, "Die reibungslose Stromung im Außengebiet zweier Kreise," Z. Angew. Math. Mech. 9, 299 (1929).

${ }^{7}$ L. Landweber and A. Shahshahan, "Added masses and forces on two bodies approaching central impact in an inviscid fluid," Technical Report No. 346 (Iowa Institute of Hydraulic Research, 1991).

${ }^{8}$ Q. X. Wang, "Interaction of two circular cylinders in inviscid fluid," Phys. Fluids 16, 4412 (2004).

${ }^{9}$ A. V. Borisov, I. S. Mamaev, and S. M. Ramodanov, "Dynamics of two interacting circular cylinders in perfect fluid," Discrete Contin. Dyn. Syst. 19, 235 (2007).

${ }^{10}$ R. S. Alassar and M. A. El-Gebeily, "Inviscid flow past two cylinders," ASME Trans. J. Fluids Eng. 131, 054501 (2009).

${ }^{11}$ E. Kanso, J. E. Marsden, C. W. Rowley, and J. B. Melli-Huber, "Locomotion of articulated bodies in a perfect fluid," J. Nonlinear Sci. 15, 255 (2005).

${ }^{12}$ D. Crowdy, "A new calculus for two-dimensional vortex dynamics," Theor. Comput. Fluid Dyn. 24, 9 (2010).

${ }^{13}$ D. Crowdy, "The spreading phase in Lighthill's model of the Weis-Fogh lift mechanism," J. Fluid Mech. 641, 195 (2009).

${ }^{14} \mathrm{~N}$. Bampalas and J. M. R. Graham, "Flow-induced forces arising during the impact of two circular cylinders," J. Fluid Mech. 616, 205 (2008).

${ }^{15}$ N. I. Muskhelishvili, Singular Integral Equations, 2nd ed. (Dover, New York, 1953).

${ }^{16}$ G. M. Goluzin, Geometric Theory of Functions of a Complex Variable (American Mathematical Society, Providence, 1969).

${ }^{17}$ D. Crowdy and A. Surana, "Contour dynamics in complex domains," J. Fluid Mech. 593, 235 (2007).

${ }^{18}$ D. Crowdy, "Explicit solution for the potential flow due to an assembly of stirrers in an inviscid fluid," J. Eng. Math. 62, 333 (2008).

${ }^{19}$ N. I. Akhiezer, Elements of the Theory of Elliptic Functions, Translation of Mathematical Monographs (American Mathematical Society, Providence, 1990), Vol. 79.

${ }^{20}$ E. B. Saff and A. B. Snider, Fundamentals of Complex Analysis, 3rd ed. (Prentice Hall, Upper Saddle River, 2003).

${ }^{21}$ L. M. Milne-Thomson, Theoretical Hydrodynamics, 5th ed. (Dover, New York, 1968).

${ }^{22}$ S. Michelin and S. G. L. Smith, "An unsteady point vortex method for coupled fluid-solid problems," Theor. Comput. Fluid Dyn. 23, 127 (2009).

${ }^{23}$ H. Lamb, Hydrodynamics, 6th ed. (Dover, New York, 1945).

${ }^{24}$ D. Crowdy and J. Marshall, "Conformal mappings between canonical multiply connected domains," Computational Methods and Function Theory 6, 59 (2006).

${ }^{25}$ D. Weihs, "The hydrodynamics of dolphin drafting," J. Biol. 3, 8 (2004). 